\title{
Correction to: Depression literacy and health-seeking attitudes in the Western Pacific region: a mixed-methods study
}

\author{
Grace W. K. Ho ${ }^{1}$ (D) D. Bressington ${ }^{1}$ - S. F. Leung ${ }^{1}$ - K. K. C. Lam ${ }^{2}$ - A. Y. M. Leung ${ }^{1,3}$ - A. Molassiotis ${ }^{1}$. J. Ligot ${ }^{4}$. \\ C. Ranoco $^{5} \cdot$ C. Sophal $^{6} \cdot$ M. Valimaki ${ }^{1}$
}

Published online: 25 June 2018

○) Springer-Verlag GmbH Germany, part of Springer Nature 2018

\section{Correction to: \\ Social Psychiatry and Psychiatric Epidemiology \\ https://doi.org/10.1007/s00127-018-1538-6}

In the original publication of this article, Acknowledgements Section was not included.

The Acknowledgements are given below. The original article has been corrected.

Acknowledgements This study was funded by the World Health Organization Western Pacific Regional Office. The authors gratefully acknowledge the support of local implementing partners at the Department of Mental Health and Substance Abuse at the Cambodia Ministry of Health, the Fiji Ministry of Health and Medical Services, and University of Santo Tomas College of Nursing.

The original article can be found online at https://doi.org/10.1007/ s00127-018-1538-6.

Grace W. K. Ho

grace.wk.ho@polyu.edu.hk

1 School of Nursing, The Hong Kong Polytechnic University, Kowloon, Hong Kong

2 Center for Health, Social Care Research, Kingston University and St. George's, University of London, London, UK

3 Centre for Gerontological Nursing, School of Nursing, The Hong Kong Polytechnic University, Kowloon, Hong Kong

4 WHO Headquarters, Geneva, Switzerland

5 College of Nursing, University of Santo Tomas, Manila, Philippines

6 Department of Mental Health and Substance Abuse, Ministry of Health, Phnom Penh, Cambodia 